\title{
Results of the Australasian (TROG) radiotherapy benchmarking exercise in preparation for participation in the PORTEC-3 trial
}

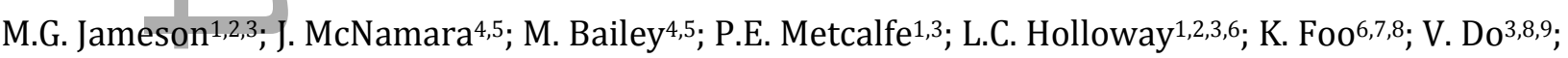

L. Mileshkin ${ }^{10,11}$; C.L. Creutzberg12; P. Khaw ${ }^{10,11}$

${ }^{1}$ Centre for Medical Radiation Physics, University of Wollongong, NSW, Australia

${ }^{2}$ Liverpool Cancer Therapy Centre, NSW, Australia

3Ingham Institute for Applied Medical Research, NSW, Australia

4 Illawarra Shoalhaven Cancer \& Haematology Network, NSW, Australia

5 Illawarra Cancer Care Centre, Wollongong, NSW, Australia

${ }^{6}$ School of Physics, University of Sydney, Sydney, NSW, Australia

${ }^{7}$ Chris O'Brien Lifehouse, Sydney, NSW, Australia

${ }^{8}$ Sydney Medical School, University of Sydney, Sydney, NSW, Australia

$15{ }^{9}$ Crown Princess Mary Cancer Centre Westmead, Sydney, NSW, Australia

10 Peter MacCallum Cancer Centre, VIC, Australia

11 University of Melbourne, VIC, Australia

12 Department of Radiation Oncology, Leiden University Medical Centre, Leiden, The Netherlands

Keywords: clinical trial, benchmarking, dummy run

Running head: PORTEC-3 Dummy Run Report

25 Corresponding author:

Michael Jameson

Cancer Therapy Centre \& Ingham Institute, Liverpool Hospital

Locked Bag 7103, LIVERPOOL NSW 1871

Australia

Phone: +402852750

This is the author manuscript accepted for publication and has undergone full peer review but has not been through the copyediting, typesetting, pagination and proofreading process, which may lead to differences between this version and the Version of Record. Please cite this article as doi: $\underline{10.1111 / 1754-9485.12447}$

This article is protected by copyright. All rights reserved 
Email: michael.jameson@sswahs.nsw.gov.au

35 Total number of pages: 9

Number of words in manuscript: 2547

Number of words in abstract: 204

Total number of tables: 1

Total number of figures: 3
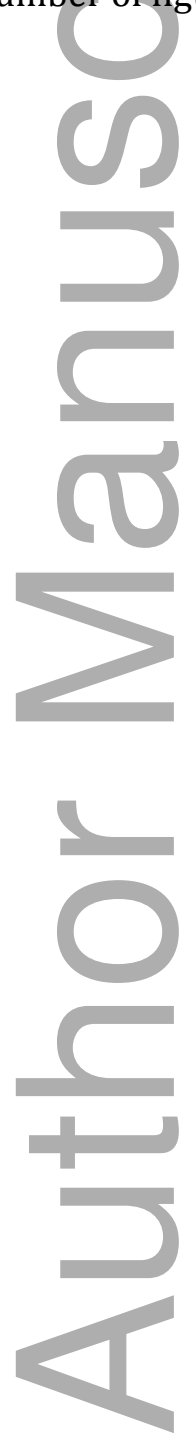

This article is protected by copyright. All rights reserved 
Received Date : 11-Nov-2015

Accepted Date : 19-Feb-2016

Article type : Radiation Oncology Original Article

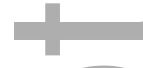

\section{Abstract}

Introduction:

Protocol deviations in Randomised Controlled Trials (RCT) have been found to result in a significant decrease in survival and local control. In some cases, the magnitude of the detrimental effect can be larger than the anticipated benefits of the interventions involved. The implementation of appropriate quality assurance of radiotherapy (QART) measures for clinical trials has been found to result in fewer deviations from protocol. This paper reports on a benchmarking study conducted in preparation for the PORTEC-3 trial in Australasia.

15 Method:

A benchmarking CT dataset was sent to each of the Australasian investigators, it was requested they contour and plan the case according to trial protocol using local treatment planning systems. These data was then sent back to TROG for collation and analysis.

Results:

20 Thirty three investigators from eighteen institutions across Australia and New Zealand took part in the study. The mean CTV volume was 383.4(228.5-497.8) $\mathrm{cm}^{3}$ and the mean dose to a reference gold standard CTV was 48.8(46.4-50.3) Gy.

Conclusions:

Although there were some large differences in the contouring of the CTV and its constituent parts,

25 these did not translate into large variations in dosimetry. Where individual investigators had deviations from the trial contouring protocol, feedback was provided. The results of this study will be used to compare with the international study QA for the PORTEC-3 trial.

\section{Introduction}

30 Accurate delineation of target volumes and organs at risk (OAR) for radiation therapy planning is required for high quality treatment as it has a direct flow-on effect for the rest of the radiotherapy chain ${ }^{1}$. Previous studies have investigated inter-observer contouring variability and the dosimetric implications on patient treatment ${ }^{1-4}$. Several methods have been suggested to improve the 
consistency of structure delineation. Training through teaching courses ${ }^{2}$ and progressive feedback such as implementing a plan-do-check-act cycle ${ }^{5}$ have been found to result in contouring submissions that are closer to the expert's delineated structures. Institutional experience gathered over the course of a trial ${ }^{6}$ and the use of atlases $^{7}$ result in more consistent submissions between observers.

40 Protocol deviations in Randomised Controlled Trials (RCT) have been found to result in a significant decrease in survival and local $\operatorname{control}^{8}$. In some cases the magnitude of the detrimental effect can be larger than the anticipated benefits of the interventions involved ${ }^{8,9}$. Implementation of appropriate quality assurance of radiotherapy (QART) measures for clinical trials has been found to result in fewer deviations from protocol ${ }^{10}$. Facility assessment prior to the trial commencing is an important aspect of QART 11 . This may include credentialing, dosimetry audits, patient case studies or a Benchmarking case $(\mathrm{s})^{12}$. In a benchmarking exercise the investigators are provided with an anonymized representative dataset and are instructed to contour and plan according to the trial protocol. A prospective evaluation is undertaken prior to trial commencement of the institution's ability to comply with the protocol and potential issues can then be fed back to the investigators. Benchmarking exercises have been used to standardize delineation procedures and have been found to increase the probability of successful completion of subsequent procedures within the same trial6.

PORTEC-3 is a RCT that compares concurrent chemoradiation and adjuvant chemotherapy with

pelvic radiation alone in high risk and advanced stage endometrial carcinoma ${ }^{13}$. Volumetric contouring for female pelvic cases was not standard practice in Australasia when PORTEC-3 opened for recruitment. Therefore, an Australasian study was implemented where investigators were required to complete credentialing before recruiting patients to the trial. Contouring variation across contributing Australasian centres is compared to a reference dataset and the impact of this variation on patient dosimetry is presented here.

\section{Materials and methods}

\section{Benchmark case procedure}

The Trans-Tasman Oncology Group (TROG) conducted a treatment planning benchmarking 65 exercise for the Australia and New Zealand Gynaecological Oncology Group (ANZGOG) PORTEC-3 RCT. This benchmarking exercise, which required clinicians involved in the PORTEC-3 study to 
contour a female pelvis according to the PORTEC-3 protocol, was not standard practice in Australia and New Zealand (ANZ) centres at the time. Thirty three observers from 18 institutions were supplied with a CD containing the anonymised DICOM images of an eligible patient. Accompanying the CD were instructions and a description of the clinical case. Once completed, this was then returned to TROG for collation and assessment by the Radiation Oncology Trial Management team.

Participants were asked to contour the case according to the PORTEC-3 protocol on their local systems. The clinical target volume(CTV) was to be delineated including the upper $50 \%$ of the vagina, the vaginal tissues superior to the vaginal marker, the paravaginal/parametrial soft tissues, and the distal common, external, and internal iliac lymph node regions. Inclusion of the subaortic pre-sacral nodes was recommended for tumours with involvement of the cervix. A margin of 7-10 mm was to be used from CTV to Planning Target Volume(PTV) with a variable margin in the upper vaginal region to account for bladder and rectal filling. Participating centres were provided with guidelines for contouring as described in the trial protocol ${ }^{13}$.

Participating institutions were also asked to plan the benchmarking case on their local treatment planning systems(TPS). The PORTEC3 protocol specified a total dose of 48.6 Gy in 1.8 Gy fractions, five times a week. Patients were to be treated on a linear accelerator using $6 \mathrm{MV}$ photons or higher. The dose was specified at the ICRU reference point and homogeneity requirements were according to ICRU-50 recommendations ${ }^{14}$. Either a 4-field (4FLD) box, 3-field or multiple field technique could be used. The treatment plan was computed using the dose grid spacing that was standard to the local hospital practice. Sites were required to export all relevant data including dose, treatment plan, DRRs and DVHs. Dose information was to be provided for CTV, PTV, both kidneys and the 90 spinal cord.

\section{Structure analysis}

A qualitative analysis was undertaken whereby a local expert panel reviewed the benchmarking case data using the SWAN ${ }^{15}$ system provided by TROG. The expert reviewers analysed the plans to 95 ensure that the target and OAR structures had been contoured per protocol. Results were categorized into acceptable, minor variation, major variation or inevaluable.

Further quantitative geometric and dosimetric analysis was performed on the submitted plans after review by the expert panel. All evaluable DICOM data was imported into Matlab using CERR ${ }^{16}$ 
where in house software ${ }^{1}$ was used to analyse geometric variation. The geometric parameters analysed included volume, centre of mass(COM), dimension( $\mathrm{x}, \mathrm{y}, \mathrm{z})$ and DICE similarity coefficient ${ }^{17}$. A consensus contour generated by the local expert trial QA group per protocol guidelines was used as a 'gold standard' reference for comparison when calculating the DICE similarity coefficient.

\section{Dosimetric analysis}

Dose volume histogram (DVH) data were extracted for the 'gold standard' target and OAR contours for each observer plan submitted, noting that these 'gold standard' contours were different to those for which the plan was generated at the local institutions (see above). The Comp Plan ${ }^{18}$ program was used to extract dose volume parameters for each of the structures (table 1).

\section{Results}

Thirty three investigators from 18 institutions submitted plans for review. From these, 495 individual target structures were manually reviewed with 435/495(87.9\%) acceptable, 49/495(9.9\%) had a minor variation and 11/495(2.2\%) with major variations (table 1). The 115 majority of the minor variations were due to incorrect PTV margin 22/49(44.9\%) largely in the required differential PTV for the vaginal vault CTV 18/49(36.7\%). Other minor variations included incorrect contouring of the parametrium CTV with respect to the pelvic sidewall 10/49(20.4\%) and contouring of the pelvic lymph nodes 11/49(24.5\%). Major variations related to PTV margin of greater than $1 \mathrm{~cm}$ being used 1/11(9.1\%) and incorrect superior CTV boundary 4/11(36.4\%) and

120 lymph node contouring 5/11(45.5\%).

Of the 33 investigator datasets, 22 were evaluable in DICOM format for quantitative geometric and dosimetric analysis, see figure 1 . The main reasons for the reduced number of datasets were corruption, duplication, incorrect format (RTOG) and missing data after transfer from review

125 software for quantitative analysis. The CTV geometric structure analysis results can be seen in figure 2. The range in volumes of contoured CTV volume was $228.5-497.8 \mathrm{~cm}^{3}$ with a mean of 383.4 $\mathrm{cm}^{3}$. The mean CTV $x$ (med/lat), $y$ (ant/post) and $z$ (sup/inf) dimensions were 15.3(13.2-16.9), $11.7(9.4-13.1)$ and $15.9(10.0-21.0) \mathrm{cm}$, respectively. The mean difference in COM between the 'gold standard' and the investigator CTVs was $1.3(0.2-2.7) \mathrm{cm}$ while the mean DICE coefficient was 0.67(0.54-0.96). 
Collated 'gold standard' CTV and small bowel DVHs are displayed in figure 3 while table 2 shows mean(range) DVH parameter values for various structures. The mean V45 for the small bowel was 29.1 \pm 4.5 Gy across all observers. The mean D98 for the CTV and PTV was $87.0 \pm 6.9 \%$ and $13579.1 \pm 8.31 \%$, respectively.

\section{Discussion}

The mean CTV DICE coefficient of $0.67 \pm 0.09$ was lower in the current study compared to the reported value from Young et $a^{19}$ of $0.77 \pm 0.03$. This may be due to the smaller number of observers $140(n=3)$ in the study by Young et al compared to that of the current study $(n=22)$, as a larger number of observers is likely to have a wider spread of contouring variation. The mean dose to the CTV and

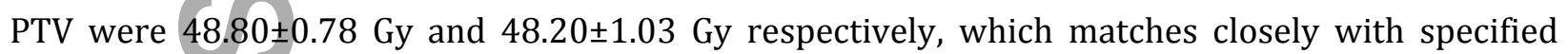
ICRU-50 reference point dose of 48.6 Gy in the trial protocol. There was however some variation in the CTV mean dose with a range of 46.44-50.30 Gy. The impact of this uncertainty in planned dose

145 is difficult to ascertain, as dose ranges of 45-50 Gy are generally found acceptable for postoperative radiation therapy in gynaecological cancers. For head and neck cancer it has been suggested that such dose variation may adversely impact the outcome of a clinical trial8.

Although the dosimetric impact of the contouring variation seen in this study was small it should be noted that the planning technique used by all centres was a simple 4FLD box. This technique is relatively insensitive to contouring variation within the borders of the "box" dose distribution. More conformal intensity modulated techniques would be more sensitive as these allow for sculpting of the dose distribution around sensitive structures such as the small bowel 20,21.

155 The participants in this benchmarking exercise were all based in Australasia and thus the results may be influenced by local treatment practices and not representative of the larger PORTEC-3 trial. Also, this benchmarking exercise was part of a credentialing activity and as such was completed at the beginning of the trial, if it were repeated later in the recruitment phase after investigators had gained additional experience the results may be different. For investigators with violations in their submitted plans, feedback was provide by the TROG trial management committee prior to enrolling patients. In order to calculate the DICE similarity coefficient, difference in COM and DVH parameters a 'gold standard' reference contour is required. The 'gold standard' in this study was a consensus volume delineated by the Australasian trial coordinators. The choice of 'gold standard' is 
controversial as there is no widely accepted technique; consensus, average, STAPLE, median and 165 simple majority have all been used previously ${ }^{1}$.

In conclusion although there were some large differences in the contouring of the CTV and its constituent parts, these did not translate into large variations in dosimetry. Where individual investigators had deviations from the trial contouring protocol, feedback was provided to ensure 170 future compliance and individual case reviews were also performed. The results of this study will be used to compare with the international study QA for the PORTEC-3 trial.

\section{References}

[1] Jameson MG, Kumar S, Vinod SK, Metcalfe PE, Holloway LC. Correlation of contouring variation 175 with modeled outcome for conformal non-small cell lung cancer radiotherapy. Radiotherapy and Oncology. 2014.

[2] Dewas S, Bibault J-E, Blanchard P, et al. Delineation in thoracic oncology: a prospective study of the effect of training on contour variability and dosimetric consequences. Radiat Oncol. 2011;6:118.

[3] Mitchell DM, Perry L, Smith S, et al. Assessing the effect of a contouring protocol on 180 postprostatectomy radiotherapy clinical target volumes and interphysician variation. International Journal of Radiation Oncology* Biology* Physics. 2009;75:990-3.

[4] Poortmans PM, Venselaar JL, Struikmans H, et al. The potential impact of treatment variations on the results of radiotherapy of the internal mammary lymph node chain: a quality-assurance report on the dummy run of EORTC Phase III randomized trial 22922/10925 in Stage I-III breast cancer. International Journal of Radiation Oncology* Biology* Physics. 2001;49:1399-408.

[5] Breunig J, Hernandez S, Lin J, et al. A system for continual quality improvement of normal tissue delineation for radiation therapy treatment planning. International Journal of Radiation Oncology* Biology* Physics. 2012;83:e703-e8.

[6] Fairchild A, Collette L, Hurkmans C, et al. Do results of the EORTC dummy run predict quality of 190 radiotherapy delivered within multicentre clinical trials? European Journal of Cancer. 2012;48:3232-9.

[7] Fuller CD, Nijkamp J, Duppen JC, et al. Prospective randomized double-blind pilot study of sitespecific consensus atlas implementation for rectal cancer target volume delineation in the 
cooperative group setting. International Journal of Radiation Oncology* Biology* Physics. 2011;79:481-9.

[8] Peters LJ, O'Sullivan B, Giralt J, et al. Critical impact of radiotherapy protocol compliance and quality in the treatment of advanced head and neck cancer: Results from TROG 02.02. Journal of Clinical Oncology. 2010;28:2996.

[9] Fairchild A, Straube W, Laurie F, Followill D. Does quality of radiation therapy predict outcomes 200 of multicenter cooperative group trials? A literature review. International Journal of Radiation Oncology* Biology* Physics. 2013;87:246-60.

[10] Bekelman JE, Deye JA, Vikram B, et al. Redesigning radiotherapy quality assurance: opportunities to develop an efficient, evidence-based system to support clinical trials-report of the National Cancer Institute Work Group on Radiotherapy Quality Assurance. International Journal of Radiation Oncology* Biology* Physics. 2012;83:782-90.

[11] Fenton PA, Hurkmans C, Gulyban A, et al. Quality assurance of the EORTC 22043-30041 trial in post-operative radiotherapy in prostate cancer: Results of the Dummy Run procedure. Radiotherapy and Oncology. 2013;107:346-51.

[12] Melidis C, Bosch WR, Izewska J, et al. Global harmonization of quality assurance naming 210 conventions in radiation therapy clinical trials. International Journal of Radiation Oncology* Biology* Physics. 2014;90:1242-9.

[13] http://clinicaltrials.gov/ct2/show/NCT00411138.

[14] ICRU Report 50: Prescribing, Recording and Reporting Photon Beam Therapy. ICRU Publ Bethesda MD. 1993.

215 [15] Ebert MA, Haworth A, Kearvell R, et al. Detailed review and analysis of complex radiotherapy clinical trial planning data: evaluation and initial experience with the SWAN software system. Radiotherapy and Oncology. 2008;86:200-10.

[16] Deasy JO, Blanco AI, Clark VH. CERR: a computational environment for radiotherapy research. Medical physics. 2003;30:979-85.

220 [17] Dice LR. Measures of the amount of ecologic association between species. Ecology. 1945;26:297-302. 
[18] Holloway LC, Miller J-A, Kumar S, Whelan BM, Vinod SK. Comp Plan: A computer program to generate dose and radiobiological metrics from dose-volume histogram files. Medical Dosimetry. 2012;37:305-9.

225 [19] Young AV, Wortham A, Wernick I, Evans A, Ennis RD. Atlas-based segmentation improves consistency and decreases time required for contouring postoperative endometrial cancer nodal volumes. International Journal of Radiation Oncology* Biology* Physics. 2011;79:943-7.

[20] Heron D, Gerszten K, Selvaraj R, et al. Conventional 3D conformal versus intensity-modulated radiotherapy for the adjuvant treatment of gynecologic malignancies: a comparative dosimetric study of dose-volume histograms ț. Gynecologic oncology. 2003;91:39-45.

[21] Small W, Mell LK, Anderson P, et al. Consensus guidelines for delineation of clinical target volume for intensity-modulated pelvic radiotherapy in postoperative treatment of endometrial and cervical cancer. International Journal of Radiation Oncology* Biology* Physics. 2008;71:428-34.

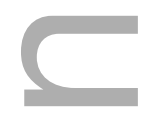

Captions:

Fig. 1. Axial, coronal and sagittal views of the benchmarking case CT displaying investigator CTVs Fig. 2. Boxplots displaying variation in contours with respect to A) volume, B) dimension, C) COM and D) DICE similarity coefficient

240 Fig. 3. Collated DVH data for all evaluable datasets for A) CTV and B) small bowel Table 1 Mean DVH parameter results for various structures (range in parentheses)

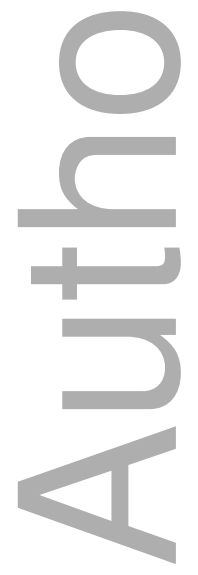


Table 1 Description of major and minor variations for the benchmarking exercise

\begin{tabular}{|c|c|c|}
\hline Variation & Structure & Description \\
\hline \multirow[t]{8}{*}{ Major } & Lymph nodes & CTV extends significantly $(>0.5 \mathrm{~cm})$ into bone \\
\hline & & Missing an iliac vessel group \\
\hline & & The CTV as contoured is not edited to exclude muscle or bowel prior \\
\hline & & to PTV expansion \\
\hline & Parametrium & Not volumed \\
\hline & CTV & Upper level $>3 \mathrm{~cm}$ inferior to the most inf part of $\mathrm{S} 1$ body \\
\hline & & Upper level $>3 \mathrm{~cm}$ inferior to the most sup part of S1 body \\
\hline & & Expansion of lymph nodes $>1 \mathrm{~cm}$; differential too large \\
\hline \multirow[t]{10}{*}{ Minor } & h nodes & External iliac nodes contoured beyond pelvis \\
\hline & & CTV significantly into muscle $(>0.5 \mathrm{~cm})$; bowel (whole loop) \\
\hline & & The CTV as contoured is not edited to exclude bone prior to PTV \\
\hline & & expansion \\
\hline & & Missing obturator strip \\
\hline & Parametrium & CTV does not reach pelvic sidewall \\
\hline & & CTV extends $<1 \mathrm{~cm}$ superior to vagina \\
\hline & Vaginal vault & CTV covers $<1 / 4$ of vagina \\
\hline & CTV & Upper level > $2 \mathrm{~cm}$ superior to most sup part of S1 body \\
\hline & PTV & Differential vault PTV margin not used \\
\hline
\end{tabular}


Table 2 Mean DVH parameter results for various structures (range in parentheses)

\begin{tabular}{lllll}
\hline & Mean (Gy) & D95\% & \multicolumn{1}{l}{ V45Gy } & \multicolumn{2}{l}{ V40Gy } \\
\hline CTV & $48.8(46.4-50.3)$ & $96.8(74.7-99.6)$ & -- & -- \\
PTV & $48.2(45.2-49.9)$ & $91.8(83.8-99.4)$ & -- & -- \\
Bladder & $46.7(37.7-49.6)$ & - & -- & -- \\
Left Femur & $29.0(19.7-34.5)$ & -- & -- & $12.43(3.0-32.6)$ \\
Right Femur & $27.1(16.4-32.7)$ & -- & -- & $9.5(1.7-27.5)$ \\
Left Kidney & $1.0(0.2-6.6)$ & -- & -- & -- \\
Right Kidney & $0.3(0.1-1.7)$ & -- & -- & -- \\
Rectum & $48.2(43.8-50.7)$ & -- & -- & -- \\
Small Bowel & $23.8(20.1-32.2)$ & -- & $29.1(19.6-38.0)$ & -- \\
\hline
\end{tabular}



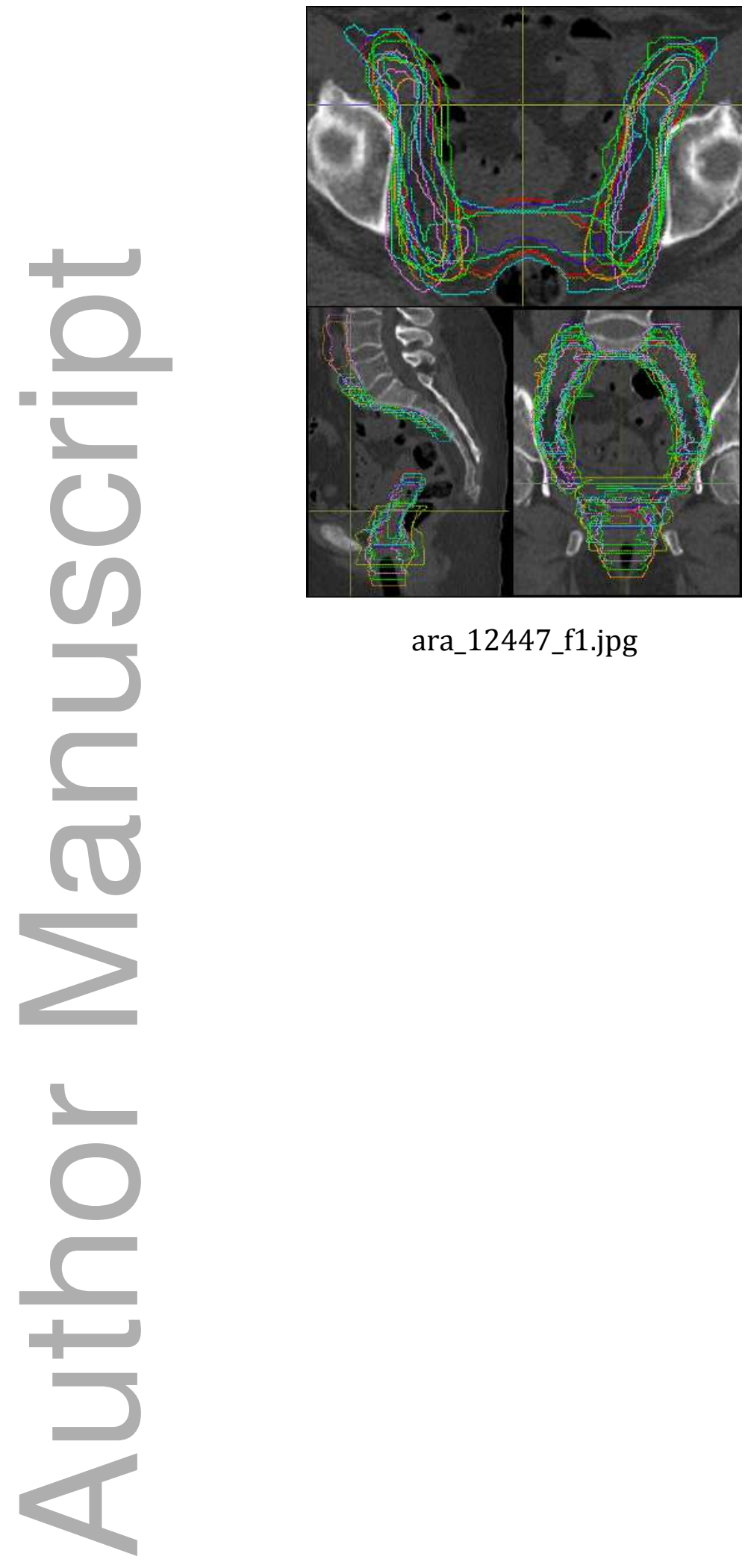

ara_12447_f1.jpg 


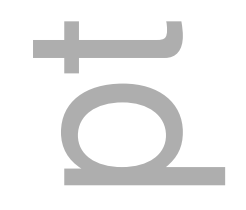

$+$
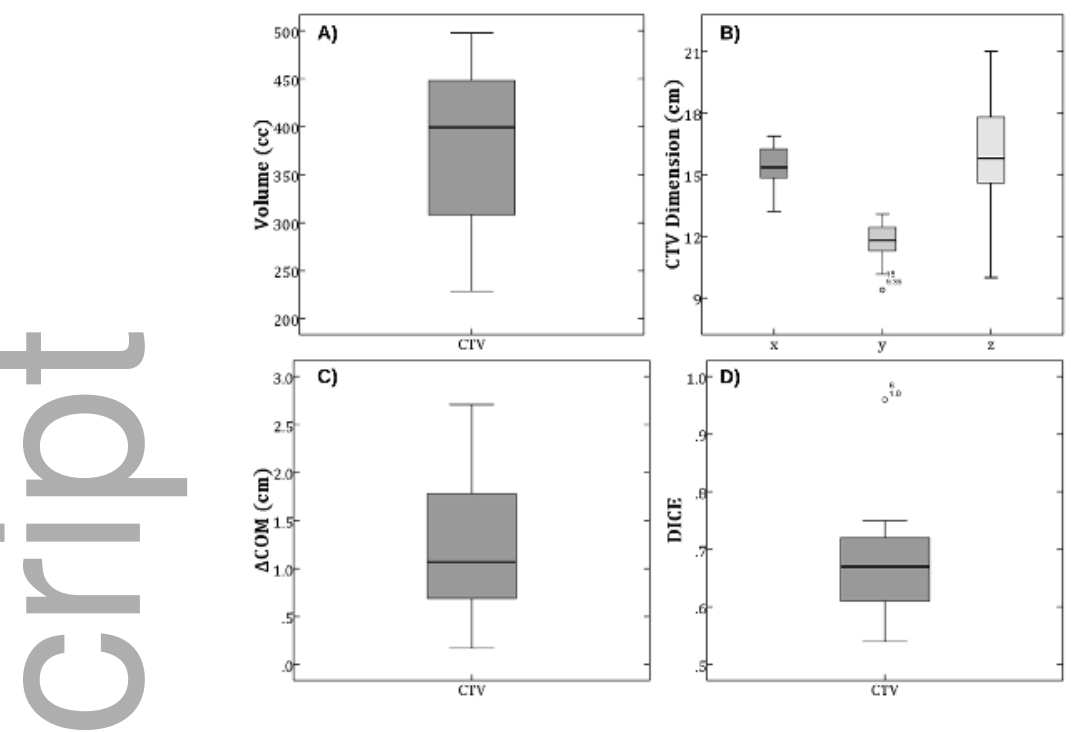

ara_12447_f2.jpg

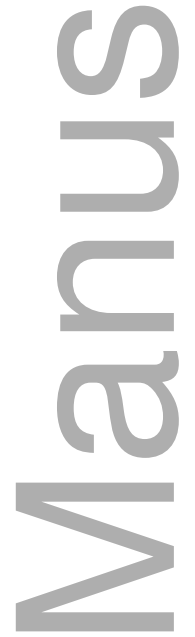

\section{ara_12447_f2.jpg}

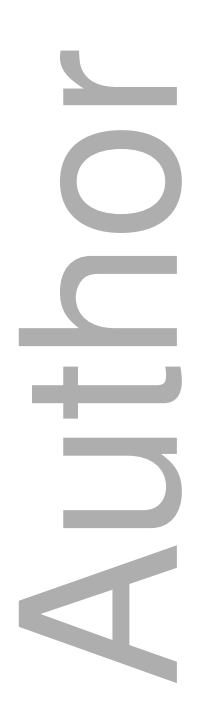



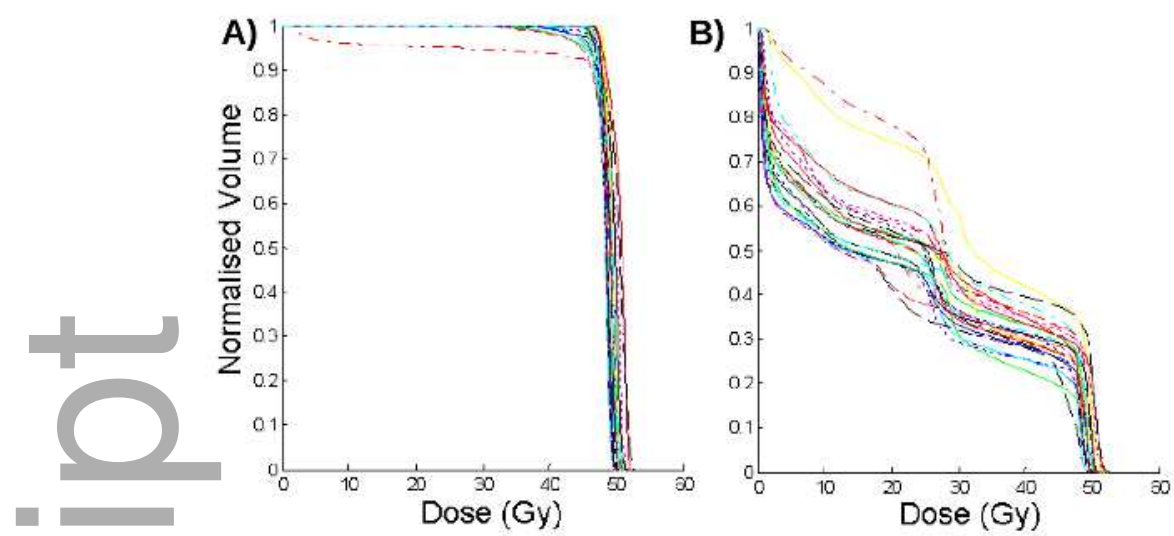

ara_12447_f3.jpg

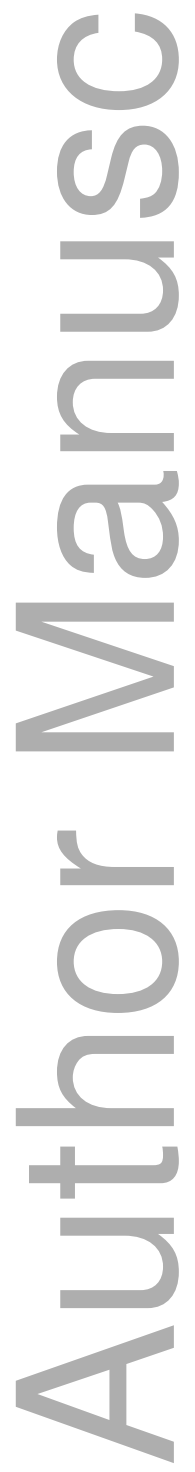

This article is protected by copyright. All rights reserved 


\section{University Library}

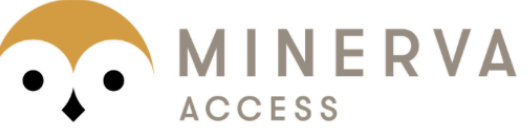

A gateway to Melbourne's research publications

Minerva Access is the Institutional Repository of The University of Melbourne

\section{Author/s:}

Jameson, MG;McNamara, J;Bailey, M;Metcalfe, PE;Holloway, LC;Foo, K;Do, V;Mileshkin, L;Creutzberg, CL;Khaw, P

Title:

Results of the Australasian (Trans-Tasman Oncology Group) radiotherapy benchmarking exercise in preparation for participation in the PORTEC-3 trial

Date:

2016-08-01

\section{Citation:}

Jameson, M. G., McNamara, J., Bailey, M., Metcalfe, P. E., Holloway, L. C., Foo, K., Do, V., Mileshkin, L., Creutzberg, C. L. \& Khaw, P. (2016). Results of the Australasian (Trans-Tasman Oncology Group) radiotherapy benchmarking exercise in preparation for participation in the PORTEC-3 trial. JOURNAL OF MEDICAL IMAGING AND RADIATION ONCOLOGY, 60 (4), pp.554-559. https://doi.org/10.1111/1754-9485.12447.

Persistent Link:

http://hdl.handle.net/11343/291146 